Ramos, P., y Holguín, H. F. (2019). Impacto de la comunicación intercultural en la competitividad para las empresas de la industria cervecera en Ciudad Juárez. Revista Lebret 11, 59 - 77. https://doi.org/10.15332/rl.v0i11.2412

Artículo de investigación:

\title{
Impacto de la comunicación intercultural en la competitividad para las empresas de la industria cervecera en Ciudad Juárez
}

\author{
Impact of intercultural communication on competitiveness for \\ beer industry companies in Juarez City.
}

\section{Patricia Ramos Rubio \\ Héctor Francisco Holguín Villalobos 2}

\begin{abstract}
Resumen
El artículo deriva de un estudio basado en la relación entre la comunicación intercultural y la competitividad en la industria cervecera, escrito bajo distintas perspectivas, tanto a nivel empresarial como a nivel cliente, para ofrecer un panorama de la relación constituida por elementos culturales, comunicativos y competitivos, con el objetivo de identificar la influencia de la comunicación intercultural para la competitividad. El trabajo se basa en un método cuantitativo con un enfoque descriptivo, correlacional y parte de la generación de nuevos factores para aumentar la competitividad de las organizaciones, que plantean un nuevo horizonte de soluciones. Los resultados muestran opiniones inversas para la sensibilidad a las actitudes y cambios en las necesidades de los clientes, resultando interesante que los valores culturales de una determinada población son conocimiento esencial para las empresas al enviar mensajes a sus clientes potenciales, además de resultar significativo el uso de estos valores para la producción de nuevos diseños de sus productos. Actualmente, la percepción de la relación entre la comunicación intercultural y la competitividad resulta ser interesante y es evidente que en la industria cervecera es fuerte.
\end{abstract}

\section{Palabras clave}

Comunicación intercultural, competitividad, Ciudad Juárez.

Códigos de clasificación JEL: M30, L81

\begin{abstract}
The article derives from a study based on the relationship between intercultural communication and competitiveness in the beer industry, conducted under different perspectives both at the business level and at the client level, offering an overview of the relationship constituted by cultural, communicative and competitive, with the objective of identifying the influence of intercultural communication for competitiveness. The study is based on a quantitative method with a descriptive, correlational approach and part of the generation of new factors to increase the competitiveness of organizations, which pose a new horizon of solutions. The results show inverse opinions for the sensitivity to attitudes and changes in the needs of their clients and it is interesting that the cultural values of a given population are essential knowledge for companies when sending their messages to their potential clients, in addition to being significant the use of these values to produce new designs of their products. Currently, the perception of the relationship between intercultural communication and competitiveness is interesting and in the beer industry it is strong.
\end{abstract}

\section{Keywords}

Intercultural communication, competitiveness, Ciudad Juárez.

1 Universidad Autónoma de Ciudad Juárez. Correo electrónico: pramos@uacj.mx Código ORCID: 0000-0003-39-79-2431

2 Universidad Autónoma de Chihuahua. Correo electrónico: Hector.holguin10@gmail.com 


\section{Introducción}

En la actualidad las empresas deben enfrentar diversas situaciones que pueden influir en continuar o no en el mercado. Aunado a esto, las extensas necesidades y exigencias de los clientes hacen que las empresas deban pensar de forma innovadora en buscar ventajas sobre las demás y así lograr posicionamiento en el mercado.

Ante este entorno hostil en el que las empresas se desenvuelven, y surge la necesidad de nuevos factores que aumenten la competitividad de estas. Tal y como lo mencionan Aznar, Bagur \& Rocafort (2015) los esfuerzos que se hacen en la actualidad para desarrollar elementos competitivos son resultado del fuerte movimiento globalizado que se ha generado en la última década en todos los ámbitos que rodean a las empresas.

Dentro de los amplios estudios en materia de competitividad, siempre se buscan maneras de que los distintos ramos que conforman la economía, encuentren factores que midan esta característica y que también busquen la manera de desarrollarla (Girán, Vargas y Osta, 2008). Por ello se considera que son los elementos inherentes al ser humano los que podrían lograr el beneficio buscado.

En un entorno general, Mas, Paluzie, Pons, Quesada, Robledo y Tirado (2007) mencionan que la competitividad es la capacidad de las organizaciones de canalizar las dificultades u oportunidades del entorno competitivo en beneficio y que permita sostener o hacer crecer los beneficios que se están percibiendo y, Lombana (2011) la conecta como un proceso de análisis de la contienda entre toda organización que ofrezca algo a la sociedad en una región, con el fin de concretar intenciones.

Estos entornos que envuelve a las empresas dificultan el crecimiento de su competitividad, por lo que es muy importante que las empresas puedan generar desde su interior nuevas oportunidades que le permitan defenderse de la competencia, a ser cada vez más innovadoras. La comunicación intercultural puede representar esa nueva oportunidad y detallar el contexto actual de la misma, es de gran valor para las empresas.

Rizo y Romeu (2006) consideran que la comunicación intercultural es aquella que tiene como base la presentación, por parte de distintos individuos que participan en un evento comunicativo, de los principios que conforman las bases culturales de cada uno y que les ayuda a aprender, y así comprender nuevos sistemas que permitan una buena interacción.

Se ha considerado a la comunicación intercultural como un posible factor que afecte la competitividad, y por ello es interesante preguntarse ¿Cuál es el impacto de la comunicación intercultural y la competitividad de las empresas de la industria cervecera en Ciudad Juárez? Y, por otra parte, analizar ¿Cuál es la influencia de la comunicación intercultural de las empresas de la industria cervecera de Ciudad Juárez para la competitividad?

Debido a la falta de factores que incrementan la competitividad, el tener un acercamiento de la influencia positiva que pueda ejercer la comunicación intercultural 
sobre esta, ofrece una innovación y beneficio para las organizaciones privadas, gubernamentales y aquellas que se dedican solamente a la investigación.

En Latinoamérica, en años recientes, el interés por esquemas integrales ha aumentado con el objetivo de progresar en la competitividad y comprender las problemáticas que la rodean. No solo México, también Brasil ha adoptado la competitividad sistémica para trabajar en direcciones particulares a través de un modelo que pondera los diferentes sectores y sus características específicas para considerar con ello estrategias y dar su debida importancia (Morales y Castellanos, 2007).

\section{Marco teórico}

México tiene múltiples elementos ventajosos para competir, además de ser un país abierto; pero en lo que respecta a la competitividad presenta una paradoja, debido a que es uno de los países menos competitivos, aun así, se destaca que en tres continentes en algunos de sus países cuenta con 31 acuerdos del libre comercio y un índice de 70\% de apertura al exterior (Villareal y Ramos, 2001).

Por otra parte, una gran cantidad de empresas en México desconocen la prioridad de desarrollar estrategias competitivas para responder a las necesidades actuales y cambiantes donde eleven su competitividad, parte de estas circunstancias ha impedido que las empresas favorezcan su desarrollo y que no se adapten en lo que respecta a los contextos tecnológicos y económicos (Ynzunza e Izar, 2013).

La competitividad es un tema cuyo desarrollo ha sido relevante en épocas recientes. Las bases que dieron partida al concepto se generan en el siglo XVII con las teorías clásicas del comercio internacional, en las que David Ricardo hablaba de las ventajas comparativas, las cuales se fundamentan en la capacidad o cantidad de recursos productivos con los que cuenta una región específica (Rojas y Sepúlveda, 1999).

Rozas \& Sánchez (2004) mencionan que el término competitividad tuvo un amplio desarrollo en la década de 1980, se le relacionaba con la capacidad, el bienestar económico de las naciones y la participación de estas en los tratados y negociaciones internacionales; cuyo objetivo principal era tomar parte importante de los mismos mercados. Procedente de los cambios del entorno internacional, surgieron nuevas teorías que se fueron extendiendo con el tiempo. Ejemplo de ello es la Teoría de Recursos y Capacidades (TRC).

que analizó el entorno competitivo predominante en los ochenta y que ha dado paso a un cambio de paradigma, el cual considera el análisis interno de la organización en especial sus recursos y capacidades como el aspecto prioritario. Ello hace que las organizaciones deban dedicar atención a identificar, desarrollar, proteger y desplegar aquellos recursos y capacidades que aseguren una ventaja competitiva sostenible, siendo esta la idea básica de dicha teoría (Suárez e Ibarra, 2002, p. 85). 
Es por lo que la competitividad desde hace varias décadas tiene una gran necesidad de innovación y de factores que impulsen su desarrollo. En la actualidad son muchos los elementos que envuelven al concepto de la competitividad y es difícil detectar aquellos que son fundamentales para su existencia. Feurer \& Chaharbaghi (1994) establecían que los factores que generan la competitividad se derivan del entorno conformado por las organizaciones, su rivalidad, y del intento de estas por ganar la mayor parte del mercado.

El enfoque de competitividad genera que los diversos sectores obtengan beneficios elevados, es aquí donde la TRC concentra su atención, asumiendo que las empresas sean diferentes, al igual que sus recursos estratégicos, por ello recomiendan estudiar el sector empresarial con el objetivo de que las compañías obtengan beneficios por encima de los normales (Arbelo y Pérez, 2001).

Por otra parte, la competencia ha crecido a nivel internacional y esto requiere cambios en los que las empresas y las regiones puedan transformar sus estructuras y ser eficientes económicamente, ante ello se hace necesario estrategias de carácter sistémico para generar y elevar la competitividad en los diferentes sectores productivos; sumando a las diferentes regiones del país generar apertura comercial (Hernández, 2006). "Las organizaciones requieren alinearse a las oportunidades y amenazas externas de la industria e implementar una estrategia que les permita generar los recursos y capacidades organizacionales que les brinden una ventaja competitiva" (Ynzunza e Izar, 2013, p. 170).

Mas, et. al. (2007) concuerdan con la dificultad de establecer el concepto competitividad en un plano regional o nacional, pero a nivel organizacional ya es fácil establecerlo; en un entorno general mencionan que la competitividad se define también como la capacidad de las organizaciones de canalizar las dificultades u oportunidades del entorno competitivo en beneficios y hacerlos crecer. De ahí la importancia de la TRC que radica en elevar las ventajas competitivas de las organizaciones evaluando sus recursos y capacidades, destacando las dimensiones y la importancia de cada una de ellas para el éxito de las empresas (Costa y Lorente, 2008). "Resulta claro que son los recursos y capacidades los que hacen que las empresas se diferencien entre sí; y la interacción entre ellos lo que convierte a la empresa en un ente único e irrepetible (Pulido, 2010, p. 4).

Apodaca, Maldonado y Máynez (2016) de la misma manera enfatizan que el grado de concientización de las capacidades como una estrategia, impacta poco en las empresas mexicanas, afectando la creación de una posición competitiva, por ello es que se destaca elevar el interés y generar información que contribuya al desarrollo del conocimiento actual. Y como lo asevera (Porter y Kramer, 2006) "Toda empresa que opera dentro de un contexto competitivo afecta significativamente su capacidad".

El enfoque, la importancia y el propósito de la TRC es identificar los recursos y su valoración por parte de las empresas para determinar sus ventajas competitivas 
y exponer que la rentabilidad de las empresas es debido a que sus componentes de éxito están sujetos a los mismos factores del sector económico (Huerta, Navas y Almodóvar, 2004).

La orientación en la competitividad de una región específica se debe fijar en los recursos que la misma región ofrece. Tal y como menciona Berumen (2006) que la competitividad de una región se genera con el esfuerzo del gobierno y sus decisiones, que inician la comunicación con las empresas que se establecen en el lugar y con todos aquellos que recopilan información del tema, teniendo como fin el bienestar social. Este proceso se observa en la figura 1.

Figura 1. Factores relacionados con la competitividad en la concreción de fines del desarrollo local

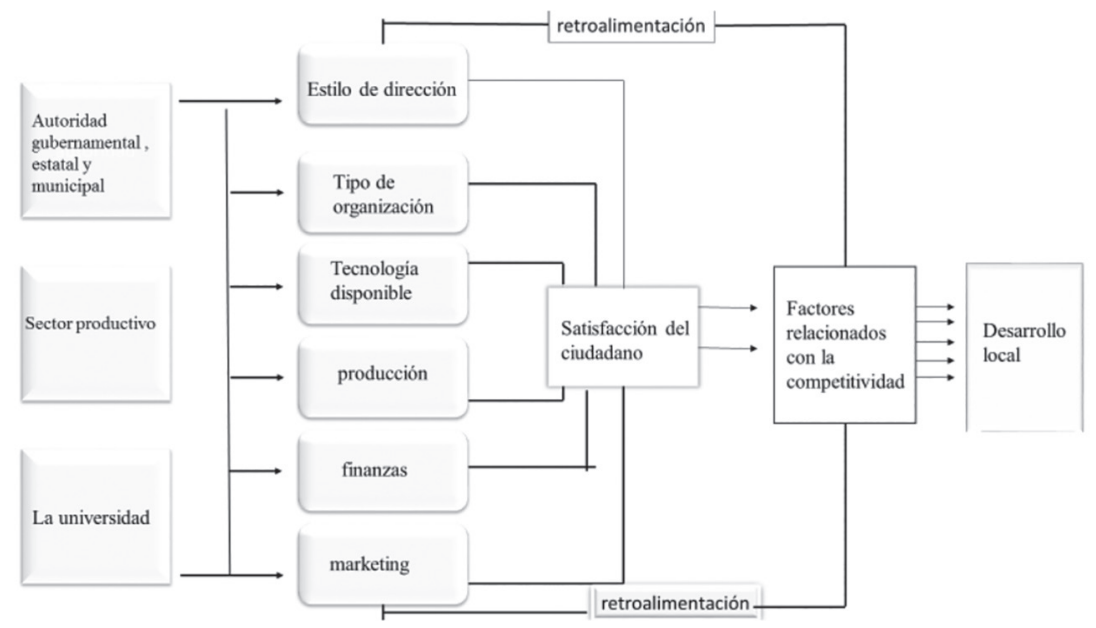

Fuente: Berumen, 2006.

La competitividad en los mercados cada día es más fuerte y la exigencia aumenta y la teoría organizacional propone que las empresas den prioridad a los factores necesarios para dar respuestas con rapidez, enfatizando como primordiales cuatro elementos: Flexibilidad; eficiencia; la sensibilidad tecnológica e innovadora que de igual manera permite elevar la competitividad de las firmas y, crear escenarios de éxito (Benavides, Muñoz y Parada, 2004).

Por otro lado, la competitividad empresarial se desarrolla desde varias perspectivas; primero, desde la competencia interna de la empresa donde se evalúa el éxito de las estrategias que se están desarrollando y también la efectividad y recurrencia con la que estas se generan en los distintos departamentos; la otra parte, es la competencia en el ambiente que rodea a la empresa, que se trabaja manejando las fortalezas para lograr aventajar a las demás compañías (Zaldívar y Gil, 2004). 
Existen también múltiples ventajas para el desarrollo regional y empresarial con la competitividad sistémica, debido a que captura determinantes políticos y económicos de éxito en las industrias y su apertura permite incluir las fortalezas y debilidades, buscando escenarios para ampliar el desarrollo industrial continuo y además de la creación de redes entre los gobiernos y las distintas empresas para elevar la competitividad nacional (Labarca, 2007).

El objetivo de la corriente de la competitividad es ampliar los mercados y buscar la evolución de las empresas a nivel regional y mundial en sus esquemas de producción, distribución y comercialización, para producir bienes y servicios altamente competitivos en todos los sentidos (Alcívar, 2015). La elaboración de estrategias es la clave para las empresas y las fuerzas competitivas, es lo que va a establecer la rentabilidad en los diversos sectores, ya que trascienden en ser el componente más importante que pueden llevar a una transformación exitosa (Porter, 2008).

Es así como la competitividad y la comunicación intercultural han tomado distintos giros a lo largo de su desarrollo y en la formulación de estrategias, pero es claro que cualquier factor relacionado con la comunicación es clave en el éxito de cualquier organización. Como menciona Spinks \& Wells (1997) la comunicación puede ser un recurso muy útil dentro de un contexto con una variedad de culturas, siempre y cuando se cuiden los aspectos que implican cada una de ellas.

El marco conceptual y factores que derivaron el contexto real de comunicación intercultural se le atribuye a Edward Hall en la década de los 50 mientras participaba en algunas investigaciones en torno a la comunicación junto con algunos otros investigadores en que se apoyaba en el FSI (Foreign Service Institute) (Rogers, Hart \& Miike, 2002). Este hecho fue el primero de muchos estudios que iniciarían el tema. El estudio formal de la comunicación intercultural y los métodos y técnicas de aplicación nace en Estados Unidos a finales del siglo XX como resultado de resolver las problemáticas comunicativas que se tenían con las personas que llegaban e interactuar con el país con modelos culturales totalmente distintos (Albut, 2012).

En la actualidad la comunicación intercultural es una herramienta muy útil en cualquier sector donde se le utilice. Por ejemplo, Pepe (2012) define la comunicación intercultural como la rama de estudio donde se cuidan los aspectos comunicativos inherentes a cada cultura, se estudian las diferencias principales entre ellas y se trata de crear modelos para servir como una herramienta moderadora donde existan variaciones de este tipo que puedan crear algún conflicto.

La comunicación siempre ha sido parte básica de la intervención entre culturas y en el desarrollo de sus relaciones. Puede ser constituida como un proceso que ayuda a compartir pensamientos, conocimientos y formas de vida, conformando enlaces entre individuos y grupos que pueden interactuar en paz gracias al diálogo y que pueden formar un constructo social sano que asegure una convivencia comunitaria positiva (Rizo, 2013). 
La teoría se extiende a una mayor cantidad de bases que están implicadas en la adopción de una nueva cultura y que también están relacionadas con la comunicación intercultural. Lo importante es que pareciera que, en el proceso de adopción de nuevos rasgos culturales, sea cual sea la teoría, la comunicación intercultural actúe como un medio de aprendizaje y de intercambio de pensamientos que llevan al crecimiento de los factores sociales y empresariales.

Aunque la competitividad y la comunicación intercultural son conceptos muy estudiados, los estudios de su relación son poco comunes. En el plano de la comunicación organizacional ya se ha visto tal relación; la importancia de la comunicación entre los miembros de las empresas se materializa en el incremento de la competitividad, apoyándose siempre de cuidar un ambiente laboral saludable (Velázquez, 2012).

La comunicación entre gerentes y empleados es un tema manejado por muchas disciplinas, pero tales investigaciones deberían ser canalizadas al ámbito de la competitividad y productividad. El hecho de mejorar la comunicación en la organización asegura el fortalecimiento de las vías de comunicación, aumentando el crecimiento de la competitividad de la misma organización (Velázquez, 2012). Aunque la comunicación es un tema relevante en el plano competitivo aún quedan huecos de investigación en el ámbito de la interculturalidad.

Dentro de la relación comunicación y competitividad ya han existido acercamientos en varios aspectos que rodean a las organizaciones. La comunicación es una herramienta que puede ayudar a mejorar, tanto la relación entre los individuos que conforman la empresa como la relación que se tiene con los clientes, además de mejorar el flujo de información que se tiene del ambiente hostil y competitivo en el que las empresas se encuentran inmersas (Domínguez y Vera , 2006).

Figura 2. Factores de competitividad y de comunicación intercultural

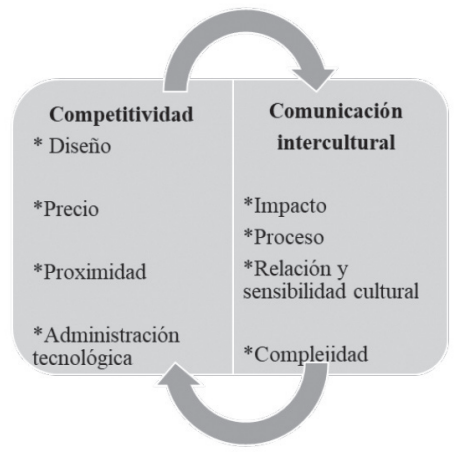

Fuente: Elaboración propia con base en (Lucato, Vieira, Vanalle y Arantes, 2012; Ricardo y Medina, 2013; Te'eni, 2001). 
Potencializar la comunicación y extenderla al ámbito intercultural puede generar beneficios extras, sobre todo si se toma en cuenta la dificultad de generar ventajas competitivas en un entorno globalizado y cambiante como el que se vive; la competitividad busca nuevos factores (figura 2) que la potencialicen y la comunicación intercultural es una gran opción, sobre todo en regiones con gran diversidad cultural, como las zonas fronterizas.

La presente investigación se basó en la industria cervecera que se desenvuelve en Ciudad Juárez. Como menciona Medina y Hernández (2013), Ciudad Juárez se caracteriza por ser una de las grandes fuentes de progreso económico que siempre ha sido adjudicado al desarrollo de la industria maquiladora, cuyo éxito se basa en su ubicación geográfica y mano de obra barata.

Bribiescas, Romero y García (2015) comentan que la relevancia de Ciudad Juárez se basa en su desarrollo industrial, siendo la ciudad más importante de Chihuahua e incluso de México en este ramo; la ciudad cuenta con bloques industriales que abarcan aproximadamente 322 empresas instaladas, pero con un crecimiento cada vez mayor por las favorables condiciones que el Gobierno y el recurso humano de la ciudad ofrecen.

Medina y Hernández (2013) con base en Maynez y Sarabia (2012) refieren que Ciudad Juárez se ubica en la frontera norte de México, colindando con Estados Unidos, lo que le ha permitido establecer buenas relaciones comerciales con dicho país y la convierte en la ciudad más importante del extenso estado de Chihuahua. Dicha ubicación y las vastas posibilidades que ofrece la ciudad han permitido que empresas de diferentes giros y nacionalidades inviertan en ella.

Por otro lado, la producción y venta de cerveza en México comenzó desde hace más de 450 años, pero no fue hasta 1890 cuando se estableció el primer negocio a nivel industrial por parte de la cervecería Cuauhtémoc; pero fue hasta mediados del siglo XX cuando la industria cervecera se fortalece en ventas y cobertura (Salomón, 2005). La industria cervecera en México siempre ha sido acaparada por pocas empresas.

Como relata Medellín (1980) desde décadas antes de su investigación, las bases de esta industria, en México, estaban controladas por tres grandes corporaciones: La Cervecería Cuauhtémoc, la Cervecería Moctezuma y la cervecería Modelo, las cuales manejaban un grupo creado por ellos mismos para el control de la producción de cebada nacional que pudiera mantener la demanda del momento.

La cerveza mexicana ha tenido gran aceptación, crecimiento y posicionamiento en los mercados internacionales debido a la gran calidad de la cebada mexicana; ejemplos de lo anterior son los del Grupo Modelo, quien al usar cebada mexicana presenta productos de mejor calidad y con un distintivo único a comparación de los de Grupo Cuauhtémoc - Moctezuma quien compra su cebada en Estados Unidos (Martínez, 2008). 
En la actualidad la producción y el mercado mexicano se mantienen en manos del Grupo Modelo y Cervecería Cuauhtémoc - Moctezuma, aunque estos fueron comprados por grandes corporativos que dominan el mercado cervecero internacional; Grupo Modelo en 2010 tenía alianza con AB InBev (quien luego lo compraría) manteniendo más del 50\% de las ventas, Cuauhtémoc - Moctezuma se unió a Heineken aumentando su participación internacional (Rojas y Rodríguez, 2010).

La industria cervecera es una industria en expansión, ya que es uno de los productos más demandados internacionalmente. La variedad de cervezas, el gran número de campañas innovadoras y las altas exigencias de los clientes hacen de esta una industria hostil en la que se requiere factores que incrementen el nivel competitivo de las empresas; por ello explorar tales factores que consigan ventajas a las empresas de este ramo es muy importante.

Como menciona (Porter, 2011) en un mundo global la competitividad se acrecienta y ello implica que las regiones asimilen y generen conocimiento, donde la cultura, la diversidad de valores, las instituciones y las estructuras económicas contribuyen al éxito de la competitividad. Actualmente, el éxito de las naciones son las industrias específicas que corresponden a entornos dinámicos y desafiantes.

\section{Metodología}

La investigación que se desarrolla es cuantitativa. Se busca describir la competitividad y la comunicación intercultural en la industria cervecera de Ciudad Juárez, mediante la recolección de datos estadísticos de encuestas a usuarios o clientes que tienen conocimiento del contexto de la industria cervecera.

La recopilación de información se realizó mediante una encuesta piloto para la validación del instrumento y su posterior aplicación definitiva. Con una población de 24.000 estudiantes, un nivel de confianza del 90\% (1.65 en valores de Z), un nivel de precisión absoluta de $95 \%$ (.05 en valor d) y valores de p y q de .5. La muestra correspondió a 269 encuestados de la Universidad Autónoma de Ciudad Juárez.

Se creó un instrumento de los factores que anteriormente fueron validados en materia de competitividad, comunicación y sus variantes culturales. Primero, se utilizó el modelo validado por Lucato, Vieira, Vanalle y Arantes (2012) quienes realizaron un recuento de diversos factores de competitividad de distintos autores, tomando estos factores y adecuándolos a la industria automotriz. Donde recomiendan hacer su revisión y adaptar los factores que se consideren para otras investigaciones.

Para efecto de esta investigación se decidió tomar como dimensiones los cuatro factores que mejor se ajustaron a la competitividad del tipo de industria por investigar, entre los que se eligió el diseño, el precio, la proximidad y la administración, realizando las respectivas modificaciones para proceder a una validación. Por otro lado, para integrar el factor comunicativo y cultural se adecuó un instrumento realizado 
por Te'eni (2001) donde formularon un modelo cognitivo-afectivo de la comunicación organizacional, conformado por tres factores principales y un cuarto factor que rodea a los tres primeros. Estos factores son el impacto, el proceso, la contribución y la complejidad de la comunicación, determinados por sus respectivas teorías, donde enfatizan la recomendación de usar este modelo para futuras investigaciones.

Dentro de esto, se tomó el factor de contribución y se modificó la relación y sensibilidad cultural con el objetivo de conjuntar la variable comunicación en relación con cultura. Finalmente, se utilizó el instrumento de Ricardo \& Medina (2013), que fue validado y en el que se realiza un estudio para analizar las actitudes y creencias acerca de la competencia intercultural entre profesores virtuales en Colombia, que manejan tres dimensiones: La conciencia del profesor de sus valores culturales, la perspectiva cultural del profesor sobre el alumno y las estrategias educativas culturalmente apropiadas que aplica el profesor.

Tabla 1. Instrumento de evaluación

\begin{tabular}{ccc}
\hline Definición conceptual & Operacionalización & Dimensiones \\
\hline $\begin{array}{c}\text { Por competitividad se considera } \\
\text { la capacidad de una empresa u } \\
\text { organización de cualquier tipo } \\
\text { para desarrollar y mantener unas } \\
\text { ventajas comparativas que le } \\
\text { permitan disfrutar y sostener una } \\
\text { posición destacada en el entorno } \\
\text { socio económico en que actúan }\end{array}$ & Competitividad & Diseño \\
& & Precio \\
\hline $\begin{array}{c}\text { La comunicación intercultural es } \\
\text { cualquier acción, manifestación o } \\
\text { proceso donde dos personas o más } \\
\text { establecen contacto comunicativo, } \\
\text { teniendo lo participantes rasgos } \\
\text { culturales distintos que son } \\
\text { adquiridos generalmente de } \\
\text { regiones geográficas también } \\
\text { distintas. }\end{array}$ & Comunicación intercultural & Pdministración tecnológica \\
& & Impacto \\
\hline
\end{tabular}

Fuente: Elaboración propia basada en Lucato, Vieira, Vanalle \& Arantes (2012); Ricardo \& Medina (2013); Te'eni (2001).

En cada dimensión se manejan distintos indicadores y preguntas, de las cuales se tomó una de la dimensión de estrategias culturalmente apropiadas, para adecuarla a la dimensión de relación y sensibilidad cultural. Para definir las variables se utilizaron las definiciones de competitividad de Zaldívar y Gil (2004, p. 65) y la de comunicación intercultural de Rizo (2013) con el fin de delimitarlas, antes de establecer las respectivas dimensiones (tabla 1). Al completar las dimensiones el cuestionario fue validado, obteniendo un Alfa de Cronbach global de 0.97 para la fiabilidad del instrumento. 


\section{Resultados descriptivos}

Se entrevistó a hombres y mujeres con el objetivo de observar una perspectiva más general e incluyente que pueda describir la comunicación intercultural y el impacto que pudiera tener sobre la competitividad.

Comenzando con los factores de competitividad, se analizó el diseño de productos que lleva la empresa, y la mayoría de los encuestados $(72.2 \%)$ están de acuerdo en que la cervecería lleva el desarrollo de nuevos diseños. Esto refleja una conexión positiva entre los consumidores y la empresa. Acerca del compromiso que tiene la cervecería con el desarrollo de nuevos productos, el 68.1\% está de acuerdo con que se asume tal obligación, el $8.5 \%$ está completamente de acuerdo, el $20.7 \%$ está en desacuerdo y el $2.2 \%$ está en total desacuerdo.

En el caso de la dimensión del precio para la competitividad, se evaluó el grado en que la cervecería toma en cuenta a los clientes para definir sus precios. Los resultados arrojan que un 55.2\% de los encuestados está de acuerdo en que se tiene en cuenta al cliente, agregando a esto un $14.4 \%$ que están totalmente de acuerdo en esta cuestión.

La proximidad que se tiene con los clientes es factor fundamental para la competitividad y al preguntar a los encuestados si la proximidad geográfica de la compañía es una ventaja sobre la competencia el $51.1 \%$ estuvo de acuerdo, mientras que un $31.5 \%$ está totalmente de acuerdo. Los porcentajes de "en desacuerdo" y "totalmente en desacuerdo" fueron bajos con $10.4 \%$ y $7 \%$, respectivamente (figura 3 ).

Figura 3. Proximidad geográfica de la compañía como ventaja competitiva

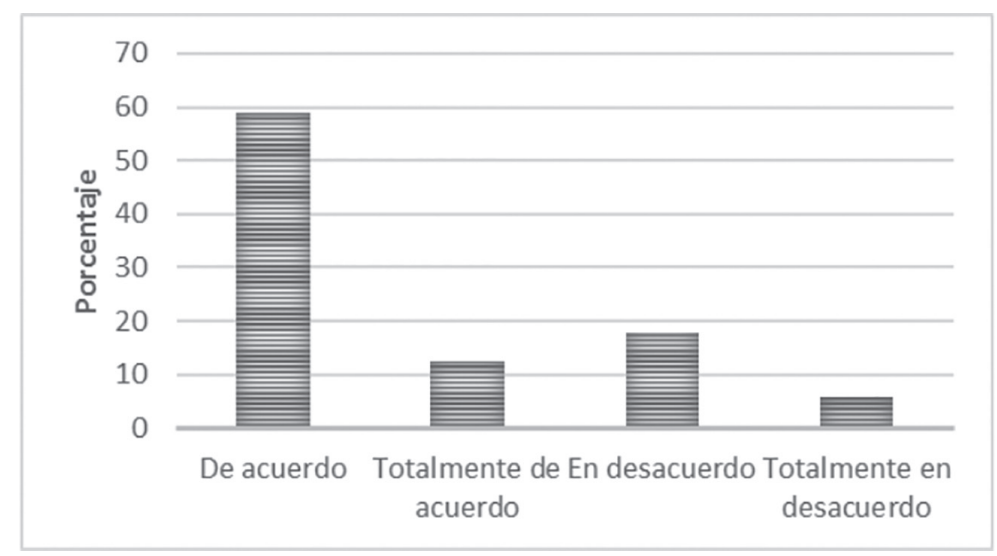

Fuente: Elaboración propia.

La última dimensión determinante de la competitividad evaluada fue la de innovaciones tecnológicas. Los resultados fueron significativos, debido a que el $60.4 \%$ de 
los encuestados está de acuerdo en que se reconoce la necesidad de que la empresa realice innovaciones tecnológicas.

En la sección del factor comunicación, respecto a la dimensión de impacto, se preguntó acerca de una verdadera y confiable comunicación entre la empresa y el cliente, los resultados obtenidos representan un choque de opiniones. Por el lado positivo, se obtuvo que un $40 \%$ está de acuerdo en que la compañía lleva tal comunicación con el cliente, aunado al 11.9\% que está totalmente de acuerdo en que se lleva esa relación. Por otro lado, el $38.9 \%$ no está de acuerdo en que se lleva una verdadera y confiable comunicación, sumado a un $4.4 \%$ que está totalmente en desacuerdo. Los resultados muestran una división de opiniones al tener porcentajes positivos y negativos casi iguales.

Continuando con la evaluación de las dimensiones de comunicación intercultural, se tienen los resultados del proceso comunicativo. Al preguntar, si creen que la compañía cuida las bases de la información que transmite, como lo es el tamaño, la distribución, la organización y la formalidad de los mensajes, el 58.5\% de ellos está de acuerdo con tal enunciado mientras que el $18.9 \%$ está totalmente de acuerdo.

Para el último factor de evaluación de la dimensión del proceso comunicativo, se evaluó si la compañía se caracteriza por tratar de impactar en el cliente al comunicarse, la mayoría, el 48.5\% de los encuestados, está de acuerdo en que sí es parte de sus características. Además, el 25.2\% está totalmente de acuerdo en que la empresa se caracteriza por tratar de impactar al cliente (tabla 2).

Tabla 2. La compañía se caracteriza por tratar de impactar en el cliente al comunicarse

\begin{tabular}{|c|c|c|c|}
\hline & & \\
\hline & & Frecuencia & Porcentaje \\
\hline \multirow{5}{*}{ Válidos } & Totalmente en desacuerdo & 18 & 6.7 \\
\hline & En desacuerdo & 38 & 14.1 \\
\hline & De acuerdo & 131 & 48.5 \\
\hline & Totalmente de acuerdo & 68 & 25.2 \\
\hline & Total & 255 & 94.4 \\
\hline Perdidos & Sistema & 15 & 5.6 \\
\hline Total & & 270 & 100 \\
\hline
\end{tabular}

Fuente: Elaboración propia.

Respecto a la dimensión de la relación y sensibilidad cultural, los resultados fueron interesantes. Al preguntar a los encuestados si creían que los valores culturales de una determinada población son conocimiento esencial para la empresa al mandar sus 
mensajes, el 54.8\% estuvo de acuerdo, mientras que el 18.1\% estuvieron totalmente de acuerdo, contra el $17 \%$ que estuvo en desacuerdo y el 4.8 totalmente en desacuerdo.

Para evaluar la complejidad de la comunicación llevada por la empresa, se les preguntó a los encuestados si creen que la compañía es sensible a las actitudes y cambios en las necesidades de los clientes, obteniendo resultados contrastantes. Por un lado, los encuestados que creen en la sensibilidad se obtuvo un $44.1 \%$ para los que están de acuerdo y un $10.7 \%$ para los que están totalmente de acuerdo, contra un $33.7 \%$ en desacuerdo y un $6.7 \%$ que están totalmente en desacuerdo.

Con la finalidad de visualizar una doble dimensión de la sensibilidad cultural inmersa en la comunicación empresa-cliente se preguntó a los encuestados si creen que la compañía es sensible a la herencia cultural propia y de sus clientes, y si trata de conservarla.

La historia cultural de la compañía a lo largo de tantos años de existencia parece reflejarse en los resultados en los que el $58.9 \%$ de los encuestados está de acuerdo con que la compañía es sensible a lo descrito y el 12.6\% está totalmente de acuerdo.

Se calculó la prueba de chi cuadrado de Pearson para la relación entre la percepción de la producción de nuevos diseños de productos por parte de la empresa, y de si los valores de determinada población son esenciales para la empresa al enviar sus mensajes, encontrándose una significancia asintótica de .002 por lo cual se demuestra una dependencia entre dichas variables (tabla 3 ).

Tabla 3. Prueba de chi cuadrado de relación de nuevos diseños y valores culturales

\begin{tabular}{cccc}
\hline & Pruebas de chi-cuadrado & & \\
\hline & Valor & Gl & Sig. Asintótica (bilateral) \\
\hline Chi-cuadrado de Pearson & $26.433^{*}$ & 9 & .002 \\
\hline Razón de verosimilitudes & 24.185 & 9 & .004 \\
\hline Asociación lineal por lineal & 12.681 & 1 & .000 \\
\hline $\mathrm{N}$ de casos válidos & 256 & & \\
\hline
\end{tabular}

Fuente: Elaboración propia.

\section{Análisis de correlación (por qué se correlacionan y cuáles factores se afectan)}

Se corrió la correlación entre los factores representativos de competitividad y los representativos de la comunicación intercultural, se presenta la tabla de correlación bivariada, (tabla 4) teniendo en cuenta solo las relaciones competitividad y comunicación intercultural, y evitando ver la relación entre los mismos factores de la variable competitividad. 
Tabla 4. Correlación bivariada entre factores de competitividad $y$ de comunicación intercultural

\begin{tabular}{|c|c|c|c|c|c|}
\hline Correlaciones & & & & & \\
\hline & & $\begin{array}{c}\text { La compañía } \\
\text { lleva el } \\
\text { desarrollo } \\
\text { de nuevos } \\
\text { diseños de } \\
\text { productos } \\
\text { para los } \\
\text { clientes }\end{array}$ & $\begin{array}{c}\text { La } \\
\text { proximidad } \\
\text { geográfica } \\
\text { de la } \\
\text { compañía es } \\
\text { una ventaja } \\
\text { competitiva } \\
\text { sobre la } \\
\text { competencia }\end{array}$ & $\begin{array}{l}\text { Se reconoce } \\
\text { la necesidad } \\
\text { de que la } \\
\text { compañía } \\
\text { realice } \\
\text { innovaciones } \\
\text { tecnológicas }\end{array}$ & $\begin{array}{c}\text { Los valores } \\
\text { culturales de } \\
\text { determinada } \\
\text { población o } \\
\text { cultura son } \\
\text { conocimiento } \\
\text { esencial para la } \\
\text { empresa al mandar } \\
\text { sus mensajes a la } \\
\text { misma }\end{array}$ \\
\hline \multirow{3}{*}{$\begin{array}{l}\text { La compañía lleva el } \\
\text { desarrollo de nuevos } \\
\text { diseños de productos } \\
\text { para los clientes }\end{array}$} & $\begin{array}{l}\text { Correlación de } \\
\text { Pearson }\end{array}$ & 1 & $.179 * *$ & .57 & $.223^{* *}$ \\
\hline & Sig. (bilateral) & & .003 & .353 & .000 \\
\hline & $\mathrm{N}$. & 270 & 270 & 270 & 256 \\
\hline \multirow{3}{*}{$\begin{array}{l}\text { La proximidad } \\
\text { geográfica de la } \\
\text { compañía es una ventaja } \\
\text { competitiva sobre la } \\
\text { competencia }\end{array}$} & $\begin{array}{l}\text { Correlación de } \\
\text { Pearson }\end{array}$ & $.179 * *$ & 1 & .067 & .237 \\
\hline & Sig. (bilateral) & .003 & & .273 & .000 \\
\hline & $\mathrm{N}$. & 270 & 270 & 270 & 256 \\
\hline \multirow{3}{*}{$\begin{array}{l}\text { Se reconoce la } \\
\text { necesidad de que la } \\
\text { compañía realice } \\
\text { innovaciones } \\
\text { tecnológicas }\end{array}$} & $\begin{array}{l}\text { Correlación de } \\
\text { Pearson }\end{array}$ & .057 & .067 & 1 & 0.75 \\
\hline & Sig. (bilateral) & .353 & .273 & & .231 \\
\hline & N. & 270 & 270 & 270 & 256 \\
\hline \multirow{3}{*}{$\begin{array}{l}\text { Los valores culturales } \\
\text { de determinada } \\
\text { población o cultura son } \\
\text { conocimiento esencial } \\
\text { para la empresa al } \\
\text { mandar sus mensajes a } \\
\text { la misma }\end{array}$} & $\begin{array}{l}\text { Correlación de } \\
\text { Pearson }\end{array}$ & $.223^{* *}$ & $.237 * *$ & .075 & 1 \\
\hline & Sig. (bilateral) & .000 & .000 & .231 & \\
\hline & N. & 256 & 256 & 256 & 256 \\
\hline ** La correlación es signi & cativa al nivel 0,0 & (bilateral) & & & \\
\hline
\end{tabular}

Fuente: Elaboración propia.

En primera instancia, la correlación bivariada entre si la compañía lleva el desarrollo de nuevos diseños de productos para los clientes y si los valores culturales de determinada población son conocimiento esencial para la empresa es significativa con un valor de .233 en la correlación de Person y una significancia de .000 permitiendo inferir que la correlación permanece constante.

También se puede observar que la correlación entre si la proximidad geográfica es una ventaja competitiva y si los valores culturales de determinada población son factor esencial al momento de enviar mensajes es significativa, ya que se tiene una correlación de Pearson de .237 y una significancia de .000 . 
Para la correlación entre el reconocimiento de la necesidad de que la compañía realice innovaciones tecnológicas y la evaluación de si los valores culturales de determinada población son conocimiento esencial para que la empresa envíe sus mensajes no es significativa, ya que presenta una correlación de Pearson de .075 y una significancia de .231 .

\section{Conclusiones}

La diversidad cultural de Ciudad Juárez es muy grande, por lo que es un reto para las empresas de la industria cervecera poder encontrar elementos que tengan mayor impacto sobre los consumidores de la región. Ante tantas estructuras culturales y una amplia gama de exigencias y necesidades distintas, la industrias en Ciudad Juárez enfrenta situaciones y posibles problemas que no se encuentran en otra región, es por ello la importancia de explorar la comunicación intercultural en la región, ya que esta eleva la competitividad de las empresas y su desarrollo empresarial.

El problema de generación de nuevos factores que aumenten la competitividad de las empresas, planteado en esta investigación, parece tener un nuevo horizonte de soluciones originadas por el ámbito comunicativo y el cultural. Actualmente, la percepción de la relación entre la comunicación intercultural y la competitividad apenas existe, pero tales variables tienen un gran peso una sobre la otra.

Las empresas presentan diferencias respecto a los cambios de sus productos para cubrir las necesidades de sus clientes, esta referencia debe tomarse en cuenta para aumentar las estrategias de comunicación intercultural. Un aspecto por resaltar en las empresas es la importancia de los valores culturales para enviar mensajes a sus clientes potenciales. Además de resultar significativo el uso de estos valores para la producción de nuevos diseños de sus productos.

Es evidente que la relación actual entre la comunicación intercultural y la competitividad en la industria cervecera es fuerte, pero aún no es captada del todo por las empresas como se puede percibir en este caso. Tal conexión llega a ser tan grande que ya es percibida de manera indirecta por los consumidores, y es identificada en el manejo de las diferentes áreas de las empresas de esta industria.

El presente trabajo describe la comunicación intercultural y la competitividad. Se destaca que en los elementos analizados los resultados sugieren que se tenga sensibilidad a las estrategias de comunicación y que se sigan teniendo en cuenta los cambios, los valores la innovación y las actitudes de los clientes para la competitividad de las empresas.

Es importante señalar que de acuerdo con los resultados la comunicación intercultural resulta ser un factor determinante para varias áreas directamente relacionadas a la competitividad, encontrando la relación cliente - empresa, los canales de comunicación entre los mismos, la profundización que la empresa hace en los 
valores culturales de los clientes, la proximidad con los clientes, el desarrollo de nuevos productos y el auge del incremento y uso de nuevas tecnologías. Lo anterior es relevante para mejorar las estrategias mercadológicas y cubrir las expectativas de los clientes. Hoy las distintas regiones y sus dinámicas requieren estrategias específicas y diferenciadas para cada uno de sus mercados, por lo que es importante este tipo de estudios para entender de una mejor manera las dinámicas de las empresas y cómo se comunican con sus consumidores.

Luego de analizar la información recopilada, se puede concluir que, efectivamente, existe una relación entre la comunicación intercultural y la competitividad en las empresas de la industria cervecera, siendo esta relación factor determinante de la conexión entre las empresas y los clientes, la manera en que estos últimos perciben los productos y ambientes, y el valor percibido en las distintas marcas.

El caso de la cervecería, es clara la descripción del desconocimiento de lo importante que pueden ser los elementos comunicativos y culturales en el desarrollo de la competitividad de las empresas. La industria cervecera, en este momento, es uno de los ejemplos de que en este tiempo los valores culturales de determinada población son clave para establecer comunicación, no solo con los clientes sino con todo el entorno que rodea a un país, lo cual en la actualidad es de gran valor.

\section{Referencias}

Albut, B. D. (2012). Intercultural communication: evolution and development issues. The Public Administration and Social Policies Revies, 1(8), 54-60.

Alcívar, A. M. (2015). Hacia la expansión del comercio internacional de la empresa local: propuesta de un plan para el estudio de la competitividad en empresas exportadoras de productos terminados ubicadas en la ciudad de Guayaquil. Anales 57, $131-141$.

Apodaca, L.E., Maldonado, S. E., y Máynez, A. I. (2016). La ventaja competitiva, desde la teoría de recursos y capacidades. Revista Internacional Administración \& Finanzas, 9(1), 69-80.

Arbelo, A., y Pérez, P. (2001). La reputación empresarial como recurso estratégico: un enfoque de recursos y capacidades. XI Congreso Nacional de Acede, Zaragoza, $1-15$.

Aznar, J., Bagur, L., y Rocafort, A. (2015). Impacto de la calidad del servicio en la competitividad y rentabilidad: El sector hotelero en la costa Catalana. Intangible Capital 12(1), 147-166. http://dx.doi.org/10.3926/ic.693

Benavides, S., Muñoz, J. J., y Parada, A. M. (2004). El enfoque de competitividad sistémica como estrategia para el mejoramiento del entorno empresarial. Economía y sociedad (24), 119-137. 
Berumen, S. A. (2006). Una aproximación a los indicadores de la competitividad local y factores de la producción. Cuadernos de Administración, 19(31), 145-163.

Bribiescas, F., Romero, I., y García, E. (2015). Turismo industrial: Oportunidades y retos dentro del sector de manufactura en Ciudad Juárez. Noésis, 24(47), 69-79. http://dx.doi.org/10.20983/noesis.2015.12.5

Costa, M. M., y Lorente, Á. M. (2008). Sistemas de gestión de calidad y resultados empresariales: una justificación desde las teorías institucional y de recursos y capacidades. Cuadernos de Economía y Dirección de la Empresa, 11(34), 7-30.

Domínguez, G., y Vera, J. (2006). Comunicación e información como generadores de competitividad. Contaduría y Administración (220), 207-229.

Feurer, R., \& Chaharbaghi, K. (1994). Defining competitiveness: A holistic approach. Management decision, 32(2), 49-58.

Girán, R., Vargas, H., y Osta, K. (2008). Propuesta metodológica para el análisis de competitividad empresarial en la pequeña y mediana agroindustria alimentaria. Revista Ingeniería Industrial, 7(1), 5-14.

Herciu, M. (2015). Challenges for business competitiveness from managerial and knowledge economy perspectives. Studies in Business \& Competitiveness, 10(3), 32-40. https://doi.org/10.1515/sbe-2015-0033

Hernández, M. (2006). La competitividad sistémica: Elemento fundamental de desarrollo regional y local. Ciencia y Mar, X(29), 39-46.

Huerta, P., Navas, J. E., y Almodóvar, P. (2004). La diversificación desde la teoría de recursos y capacidades. Cuadernos de Estudios Empresariales (14), 87-104.

Labarca, N. (2007). Consideraciones teóricas de la competitividad empresarial. Omnia, 13(2), 158-184.

Lombana, J. (2011). Looking for a distinctive model with which to analyze competitiveness. Journal of Competitiveness Studies, $19(3$ \& 4), 32-44.

Lucato, W. C., Vieira, M., Vanalle, R., y Arantes, J. A. (s.f.). Model to measure the degree of competitiveness for auto parts manufacturing companies. International Journal of Production Research 50(19),1-15.

Martínez, A. (2008). Tequila, mezcal y cerveza: De México para el mundo. Agricultura Sociedad y Desarrollo, 5(2), 143-150.

Martínez, M., y Martínez, Á. R. (2008). Sistemas de gestión de calidad y resultados empresariales: una justificación desde las teorías institucional y de recursos y capacidades. Cuadernos de Economía y Dirección de la Empresa, 11(34), 7-30. https://doi.org/10.1016/S1138-5758(08)70051-3 
Mas, M., Paluzie, E., Pons, J., Quesada, J., Robledo, J. C., y Tirado, D. A. (2007). Competitividad, crecimiento y capitalización de las regiones españolas. Dirigido por E. Reig. Fundación BBVA, Bilbao, España.

Maynez, A., y Sarabia, C. (2012). Ciudad Juárez y El Paso: Vecinos cercanos por localización, ¿Socios estratégicos en el desarrollo competitivo de la región? Colegio de la Frontera Norte. Plan Estratégico y Transversal de Ciencia y Tecnología para el Desarrollo de la Frontera Norte.

Medellín, R. (1980). Los campesinos cebaderos y la industria cervecera en México. Comercio Exterior, 30(9), 927-936.

Medina, R., y Hernández, I. (2013). Orientación al mercado y ventaja competitiva: Análisis en el contexto hotelero de Ciudad Juárez, Chihuahua. Revista Iberoamericana de Turismo, 3(2), 35-43.

Morales, M. E., y Castellanos, Ó. F. (2007). Estrategias para el fortalecimiento de las Pyme de base tecnológica a partir del enfoque de competitividad sistémica. Innovar. Revista de Ciencias Administrativas y Sociales, 17(29), 115-136.

Pepe, A. I. (2012). Comunicación intercultural en la organización. Dixit (17), 28-39. https://doi.org/10.22235/d.v0i17.354

Porter, M. (2008). Las cinco fuerzas competitivas que le dan forma a la estrategia. Harvard Business Review, 86(1), 58-77.

Porter, M. (2011). Competitive advantage of nations: creating and sustaining superior performance. Simon and Schuster.

Porter, M., y Kramer, M. (2006). Estrategia y sociedad. Harvard Business Review, 84(12), 42-56.

Pulido, B. (2010). Teoría de los recursos y capacidades: el foco estratégico centrado en el interior de la organización. Sotavento MBA (15), 54-61.

Ricardo, C. T., y Medina, A. (2013). Actitudes y creencias de la competencia intercultural en profesores virtuales. Revista Cientifica Ingeniería y Desarrollo, 31(2), 272-290.

Rizo, M. (2013). Comunicación e interculturalidad. Reflexiones en torno a una relación indisoluble. Global Media Journal México, 10(19), 26-42.

Rizo, M., y Romeu, V. (2006). Cultura y comunicación intercultural. Aproximaciones culturales. Revista da Associação Nacional dos Programas de Pós-Graduação em Comunicação, 1-19. https://doi.org/10.30962/ec.85 
Rogers, M., Hart, B., \& Miike, Y. (2002). Edward T. Hall and The History of Intercultural Communication: The United States and Japan. Keio Communication Review (24), 3-26.

Rojas, J., y Rodríguez, R. (2010). Venta de cervecería Cuauhtémoc Moctezuma, la hegemonía de la inversión extranjera directa. Economía Actual. Revista Trimestral de Análisis de Coyuntura Económica, 3(1), 6-9.

Rojas, P., y Sepúlveda, S. (1999). ¿Qué es la competitividad? San Jóse: IICA.

Rozas, P., y Sánchez, R. (2004). Desarrollo de infraestructura y crecimiento económico: revisión conceptual. Serie Recursos Naturales e Infraestructura (75), 1-75. Santiago de Chile: CEPAL.

Salomón, A. (2005). La industria de bebidas alcohólicas en México. Comercio Exterior 55(12), 1098-1104.

Spinks, N., \& Wells, B. (1997). Intercultural communication: a key element in global strategies. Career Development International, 2(6), 287-292.

Suárez, J., y Ibarra, S. (2002). La teoría de los recursos y las capacidades: un enfoque actual en la estrategia empresarial. Anales de Estudios Económicos y Empresariales (15), 63-89.

Te'eni, D. (2001). Review: A Cognitive Effective Model of Organizational Communication for Designing IT. MIS Quarterly 2(25), 251-312.

Velázquez, G. (2012). Impacto de la comunicación organizacional en la competitividad y la vinculación de las empresas mexicanas con la universidad. Recherches en Sciences de Gestion 3(90), 89-119.

Villareal, R., y Ramos, R. (2001). La apertura de México y la paradoja de la competitividad: hacia un modelo de competitividad sistémica. Comercio Exterior, 51(9), 772-788

Ynzunza, C. B., e Izar, J. M. (2013). Efecto de las estrategias competitivas y los recursos y capacidades orientados al mercado sobre el crecimiento de las organizaciones. Contaduría y Administración, 58(1), 169-197. https://doi. org/10.1016/S0186-1042(13)71202-6

Zaldívar, M., y Gil, I. (2004). Interacción entre competitividad empresarial y desarrollo local en las condiciones de economía globalizada. Economía y Desarrollo, 138(2), 55-82. 